\title{
Magnetized Plasma in Polar Semiconductors
}

\author{
A. WYSMOEEK \\ Institute of Experimental Physics, Warsaw University \\ Hoża 69, Warsaw, Poland \\ and Grenoble High Magnetic Field Laboratory, CNRS \\ 38042 Grenoble, France
}

\begin{abstract}
Plasma excitations in metallic $n$-type GaAs are studied in high-magnetic fields using the method of inelastic light scattering (the Raman scattering). Experimental data are analyzed using a standard, dielectric function theory. The results obtained for samples with a high electron concentration are well understood in terms of longitudinal excitations. A strong interaction of coupled longitudinal optical-phonon-plasmon modes with the collective cyclotron resonance excitations (the Bernstein modes) is observed. In samples with a lower electron concentration, the unexpected feature in the vicinity of the undressed optical phonon is observed at high magnetic fields. This effect is explained in terms of transverse excitations, which would appear in the Raman spectrum due to disorder-activated selection-rule breaking. A field induced metal-insulator transition and magnetopolaron effect on shallow donors in GaAs is shown to be traced with the Raman scattering experiments in samples with the lowest electron concentration.
\end{abstract}

PACS numbers: 78.30.Fs, 73.20.Mf, 78.20.Ls

\section{Introduction}

Plasma excitations in solids were intensively studied already at the time of the 1960's [1]. A significant interest was focused on doped polar semiconductors. In these systems, the effect of coupling between plasmons and longitudinal optical (LO) phonon modes can be clearly observed as demonstrated, for example, by the pioneering work of Mooradian and Wright [2] on inelastic light scattering in $n$-type GaAs samples. The interaction between LO-phonons and plasmons occurs via "macroscopic" longitudinal electric fields produced by both excitations, resulting in new, combined proper modes of the system [3]. In contrast to the infrared spectroscopy which probes the transverse and small wave-vector excitations, the 
inelastic light scattering is well adopted to study the longitudinal excitations and advantageous to probing the excitations with relatively large wave-vectors [4-7].

The properties of a plasma subjected to a static magnetic field are significantly modified $[1,8]$. A magnetic field $B$ applied to a metallic system introduces a characteristic frequency, namely the cyclotron frequency $\omega_{\mathrm{c}}=e B / m$ of motion of the carriers (with an effective mass $m$ ) in the plane perpendicular to the field direction. In polar-semiconductor plasmas, by changing the magnetic field the cyclotron frequency $\omega_{\mathrm{c}}$ can be made comparable to all important frequencies of the system, namely the plasma frequency $\omega_{\mathrm{p}}$ and the frequencies of longitudinal (LO) and transverse (TO) optical phonons. The physics of coupled plasmon-LO-phonon modes under these particular and interesting conditions has never been fully explored in the Raman scattering experiments in 3D systems, although to a large extent explored theoretically $[1,9]$.

In this paper magneto-Raman scattering studies of coupled plasmon-LO-phonon modes in bulk, $n$-type GaAs samples with different electron concentrations, ranging from $6 \times 10^{16} \mathrm{~cm}^{-3}$ up to $1.3 \times 10^{18} \mathrm{~cm}^{-3}$, are presented. Experiments were performed in a wide range of magnetic fields up to $28 \mathrm{~T}$, which cover the limit of cyclotron frequency exceeding the LO frequency of the insulating material. These studies are complementary to the more common, far-infrared absorption investigations of such effects [10-17]. However, the latter experiments probe the transverse plasma excitations which, in addition, are characterized by very small $k$-vectors provided by far infrared light. The Raman scattering spectra are sensitive to longitudinal modes and to nonlocal effects (characteristic of excitations with large $k$-vectors), and indeed offer extra information. This was already shown in the past, though the experiments reported on bulk semiconductors were restricted to relatively low magnetic fields and focused on a nondegenerate, Maxwellian plasma $[18,19]$. On the other hand, there exist a number of works, concerning both the Raman scattering [7, 20, 21] and far-infrared magneto-spectroscopy [22, 23] which address the issue of coupling between the electronic and phonon excitations in two-dimensional semiconductor structures [24]. The physics of low energy excitations in these structures is far more complex because of the modification of the energy spectrum due to effects of spatial confinement. Therefore, the studies of bulk material presented in this paper may help in a better understanding of low dimensional systems.

\section{Experimental details}

In order to study inelastic light scattering in high magnetic fields, provided by resistive magnets at the Grenoble High Magnetic Field Laboratory, a specially designed fiber optic system, with one fiber for the excitation and the second one for the collection, have to be used to transmit the light to and from the sample. A similar system was already used for selective excitation of donor acceptor-pair luminescence in GaAs, in which the high rejection rate of the laser light and Raman 
signal from the fiber is also important [25]. The experiments were performed in magnetic fields up to $28 \mathrm{~T}$ at liquid nitrogen $(T=77 \mathrm{~K})$ and helium $(4.2 \mathrm{~K})$ bath temperatures, in a backscattering geometry: the wave vector $k_{\mathrm{i}}$ of the incident light anti-parallel to the wave vector $k_{\mathrm{s}}$ of the scattered light. A tunable Ti:sapphire laser was used as the excitation source, while the signal was dispersed with a triple grating spectrometer equipped with a CCD camera. From the point of view of the effective excitation of the semiconductor plasma, a proper choice of the exciting light wavelength is very important. Using the light wavelengths from the range of 820-860 nm allowed penetrating a large volume of the crystal and therefore avoiding the signals from the surface depletion layer.

\section{Zero field Raman spectra on $n$-type GaAs}

The zero-field Raman scattering spectra, obtained for samples with different electron concentrations from $6 \times 10^{16} \mathrm{~cm}^{-3}$ up to $1.3 \times 10^{18} \mathrm{~cm}^{-3}$, are presented in Fig. 1. The Raman scattering spectra of all samples show a sharp peak associated with the TO phonon mode, which appears at the energy of $33.7 \mathrm{meV}$ characteristic of the undoped material. None of the spectrum displays a Raman signal related to the LO phonon mode of undoped GaAs. Instead, this mode, in our heavily doped samples, is replaced by coupled plasmon-phonon modes: $\omega^{-}$observed below the TO frequency and $\omega^{+}$observed above the LO frequency. As expected, $\omega^{-}$ and $\omega^{+}$frequencies depend on electron concentration $n$, or in other words on the plasma frequency $\omega_{\mathrm{p}}=\left(n e^{2} / \varepsilon_{0} \varepsilon_{\infty} m^{*}\right)^{1 / 2}$ (where $\varepsilon_{\infty}$ is the high-frequency dielectric constant and $m^{*}$ denotes the electron effective mass). Each $\omega^{-}$and $\omega^{+}$

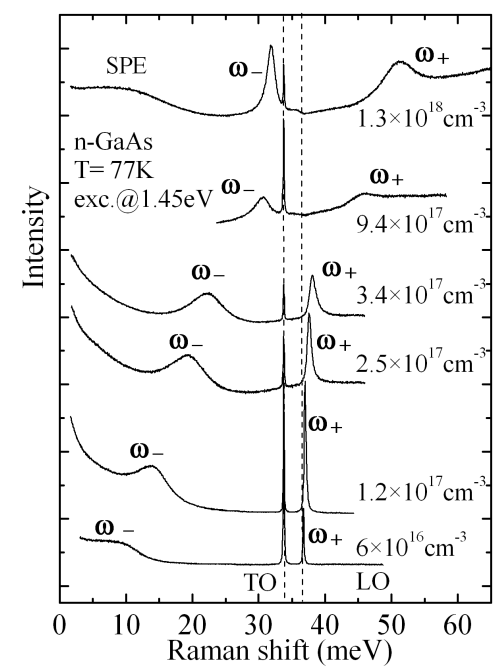

Fig. 1. The Raman scattering spectra measured for $n$-type GaAs samples with different concentrations (as shown in the figure) in the absence of the magnetic field at liquid nitrogen temperature and laser excitation energy of $1.45 \mathrm{eV}$. 
modes is characterised by its own dispersion relation, but in the limit of long wave vectors, $k \approx 0$, the corresponding frequencies are given by the following formula [3]:

$$
2\left(\omega_{ \pm}\right)^{2}=\omega_{\mathrm{p}}^{2}+\omega_{\mathrm{LO}}^{2} \pm\left[\left(\omega_{\mathrm{p}}^{2}+\omega_{\mathrm{LO}}^{2}\right)^{2}-4 \omega_{\mathrm{p}}^{2} \omega_{\mathrm{TO}}^{2}\right]^{1 / 2}
$$

Here, $\omega_{\mathrm{LO}}$ and $\omega_{\mathrm{TO}}$ are, respectively, the LO and TO phonon frequencies of an undoped material.

It is worth noticing that in a simple approximation, if the effective mass is known and does not depend on the electron concentration, i.e., the effect of nonparabolicity is neglected, a knowledge of the energies of $\omega^{-}$and $\omega^{+}$modes provides a very direct estimate of the electron concentration in the sample [26].

The plasmon- or phonon-like character of the coupled modes changes with electron concentration. $\omega^{-}$can be considered as a plasmon-like and $\omega^{+}$as a phonon-like mode at low electron concentrations, when $\omega_{\mathrm{p}} \ll \omega_{\mathrm{LO}}$. In contrast, $\omega^{-}$is phonon-like, whereas $\omega^{+}$is plasmon-like, when $\omega_{\mathrm{p}} \gg \omega_{\mathrm{LO}}$, i.e., in the high concentration limit. The plasmon- or phonon-like character of coupled modes can be clearly seen in the spectral broadening of the associated Raman scattering signals (see Fig. 1): phonon-like peaks are much narrower when compared to plasmon-like ones. As shown in the next sections, the application of magnetic field influences mostly a "plasmonic" part of the coupled plasmon-phonon modes.

\section{Limit of high electron concentrations}

The application of a magnetic field modifies the measured Raman response, and the effect is remarkably dependent on the configuration of the incident and scattered wave vectors (correspondingly, $k_{\mathrm{i}}$ and $k_{\mathrm{s}}$ ) with respect to the magnetic field $(B)$ direction [26, 27]. Except the effect of field-dependent spectral broadening, the coupled plasmon-phonon modes are little influenced by the magnetic field applied in the backscattering Faraday configuration (see Fig. 2). This is to be expected for scattering experiments as they predominantly probe the longitudinal excitations which remain unchanged when propagating along the magnetic field.

In contrast, the scattering signal from coupled plasmon-phonon modes shows more pronounced changes, both in spectral position and broadening, for the other $\left(k_{\mathrm{i}}, k_{\mathrm{s}}\right)-B$ configurations. This simply reflects the anisotropic character of the Lorentz force, which acts on the mobile charges in a magnetic field [26]. The Raman spectra measured versus magnetic field for two samples with high electron concentrations are shown in Fig. 3.

Roughly speaking, the magnetic field behaviour of the Raman scattering spectra can be understood in terms of the observation of hybrid cyclotron-plasmon-LO-phonon modes, shown with dotted lines in Fig. 4, according to the following formula [28]:

$$
2\left(\omega_{\mathrm{hyb}}^{ \pm}\right)^{2}=\Omega_{\mathrm{c}}^{2}+\omega_{\mathrm{LO}}^{2} \pm\left[\left(\Omega_{\mathrm{c}}^{2}+\omega_{\mathrm{LO}}^{2}\right)^{2}-4\left(\omega_{\mathrm{c}}^{2} \omega_{\mathrm{LO}}^{2}+\omega_{\mathrm{p}}^{2} \omega_{\mathrm{TO}}^{2}\right)\right]^{1 / 2},
$$

where $\Omega_{\mathrm{c}}^{2}=\omega_{\mathrm{c}}^{2}+\omega_{\mathrm{p}}^{2}$ and $\omega_{\mathrm{c}}$ is the cyclotron frequency. 


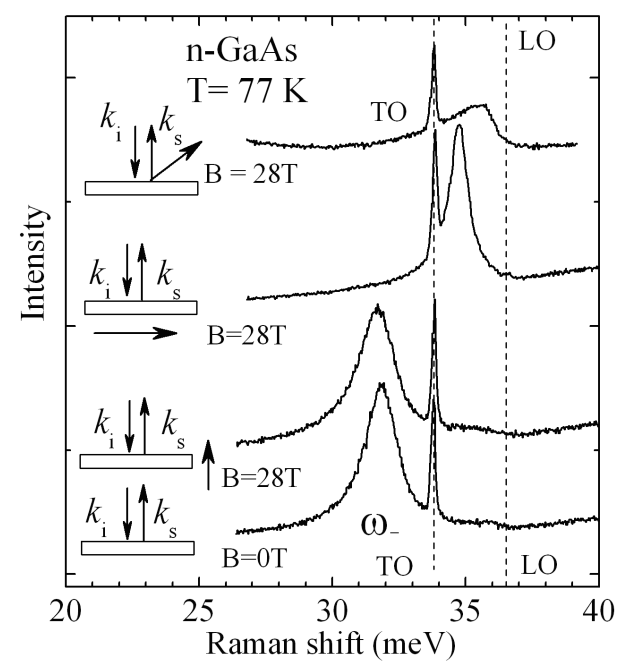

Fig. 2. Inelastic light scattering spectra measured at magnetic field $B=28 \mathrm{~T}$ in Faraday, Voigt, and oblique angle configurations on the sample with $n=1.3 \times 10^{18} \mathrm{~cm}^{-3}$. The spectrum at zero magnetic fields (bottom trace) is shown for comparison.
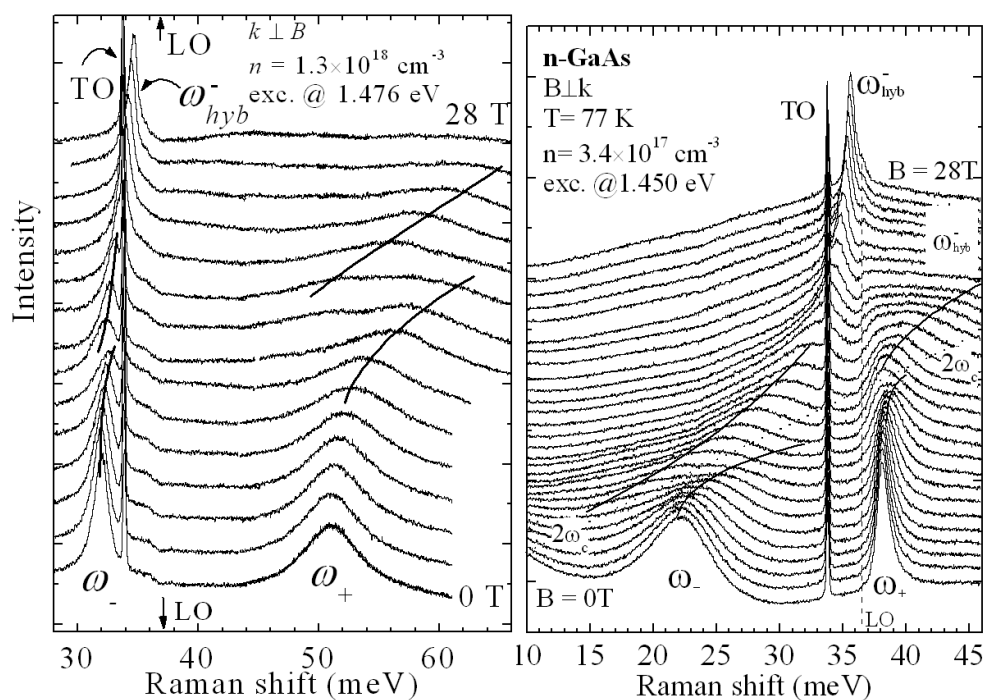

Fig. 3. Magnetic field evolution of Raman scattering spectra measured in Voigt configuration for the sample with electron concentration of $n=1.3 \times 10^{18} \mathrm{~cm}^{-3}$ (left part), $3.4 \times 10^{17} \mathrm{~cm}^{-3}$ (right part).

Upon increasing magnetic field, $\omega_{\text {hyb }}^{-}$and $\omega_{\text {hyb }}^{+}$shift towards higher energies. Around $20 \mathrm{~T} \omega_{\text {hyb }}^{-}$crosses the TO mode and eventually approaches the energy of the undressed LO phonon of undoped material. In the high field limit, $\omega_{\text {hyb }}^{-}$ 


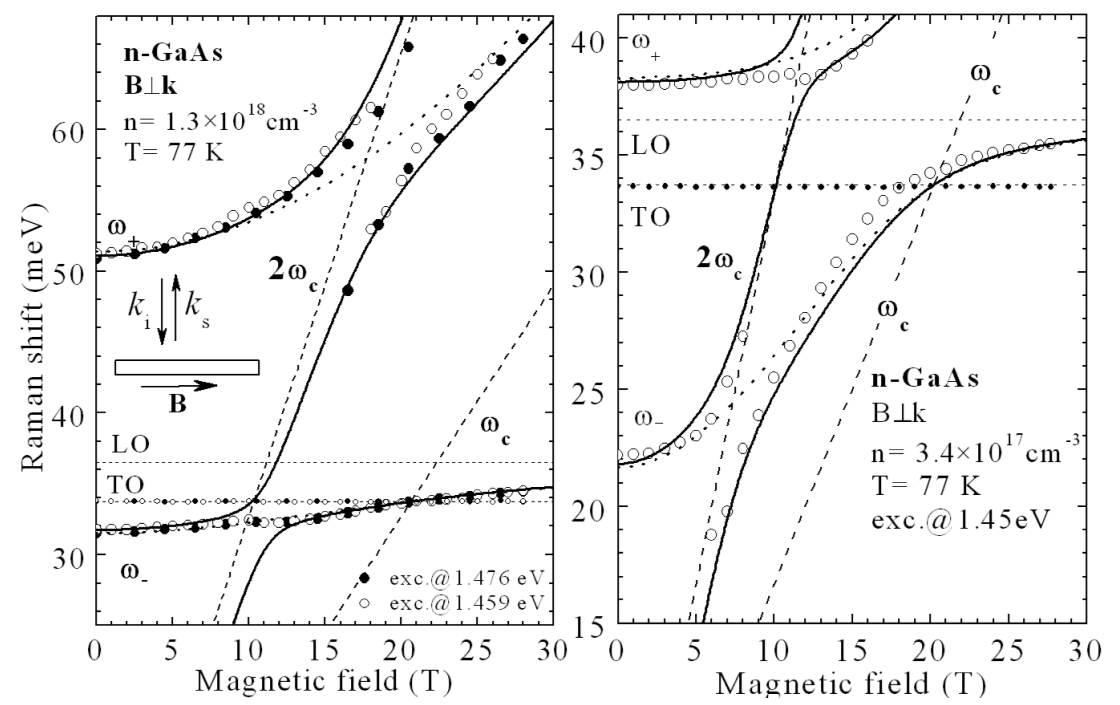

Fig. 4. The magnetic field dependence of energy positions of Raman scattering peaks measured in Voigt configuration for the sample with electron concentration of $n=$ $1.3 \times 10^{18} \mathrm{~cm}^{-3}$ (left part) and $n=3.4 \times 10^{17} \mathrm{~cm}^{-3}$ (right part). Dotted lines represent hybrid modes. Solid lines correspond to the calculation including interaction with Bernstein modes.

is getting narrower which indicates the increase in its phonon-like character at high magnetic fields. When the magnetic field tends to zero, $\omega_{\text {hyb }}^{+} \rightarrow \omega^{+}$and $\omega_{\text {hyb }}^{-} \rightarrow \omega^{-}$, whereas $\omega_{\text {hyb }}^{+} \rightarrow \omega_{\text {c }}$ and $\omega_{\text {hyb }}^{-} \rightarrow \omega_{\text {LO }}$ in the high field limit. Magnetic field dependence of the hybrid modes roughly reproduces the experimental data but clearly disregards the anticrossing effect which is particularly pronounced for the $\omega_{\text {hyb }}^{+}$mode for the sample with $n=1.3 \times 10^{18} \mathrm{~cm}^{-3}$, and for the $\omega_{\text {hyb }}^{-}$the sample with $n=3.4 \times 10^{17} \mathrm{~cm}^{-3}$. This behaviour results from interaction between multiple (collective) cyclotron excitations, called the Bernstein modes [29] and finite $k$-vector magnetoplasmon-phonon modes. The Bernstein modes are usually inactive in the Raman scattering experiments on three-dimensional plasmas except the anticrossing region when they couple with hybrid magnetoplasmon modes [19]. The detailed analysis of the Raman scattering data obtained for GaAs samples of different electron concentrations, including nonlocal effects responsible for the interaction with finite $k$-vector modes is presented in Ref. [26]. The experimental data obtained for samples, within the wide range of electron concentrations, can be reasonably well described using the standard approach of the longitudinal dielectric function but including nonlocal effects. Thus, one concludes that the model developed for the case of classical magnetoplasmas accounts well for experimental results even in the limit of high magnetic fields. However, as will be discussed later, some disagreement progressively appears when samples with lower electron concentrations are considered. 


\section{Intermediate electron concentration}

The Raman scattering spectra measured at $T=77 \mathrm{~K}$ and $T=4.2 \mathrm{~K}$ in a Voigt configuration on the sample with an electron concentration $n=2.5 \times$ $10^{17} \mathrm{~cm}^{-3}$ are shown in Fig. 5. The characteristic longitudinal plasmon-phonon excitations can be still recognized in this case: the $\omega_{\text {hyb }}^{-}$mode is particularly well pronounced at high magnetic fields. The data are more clear at $T=77 \mathrm{~K}$ than at $T=4.2 \mathrm{~K}$ because strong luminescence at low temperatures in this sample overlaps with the Raman scattering signal. The prominent feature present in these data is an additional, Raman scattering peak which develops from the low energy wing of the $\omega^{+}$mode and approaches, at high magnetic fields, the expected position of the LO mode of an undoped crystal. It appears to be clearer at lower electron concentrations and is particularly well pronounced in the low temperature data, when localization effects begin to play a role (see Fig. 5).
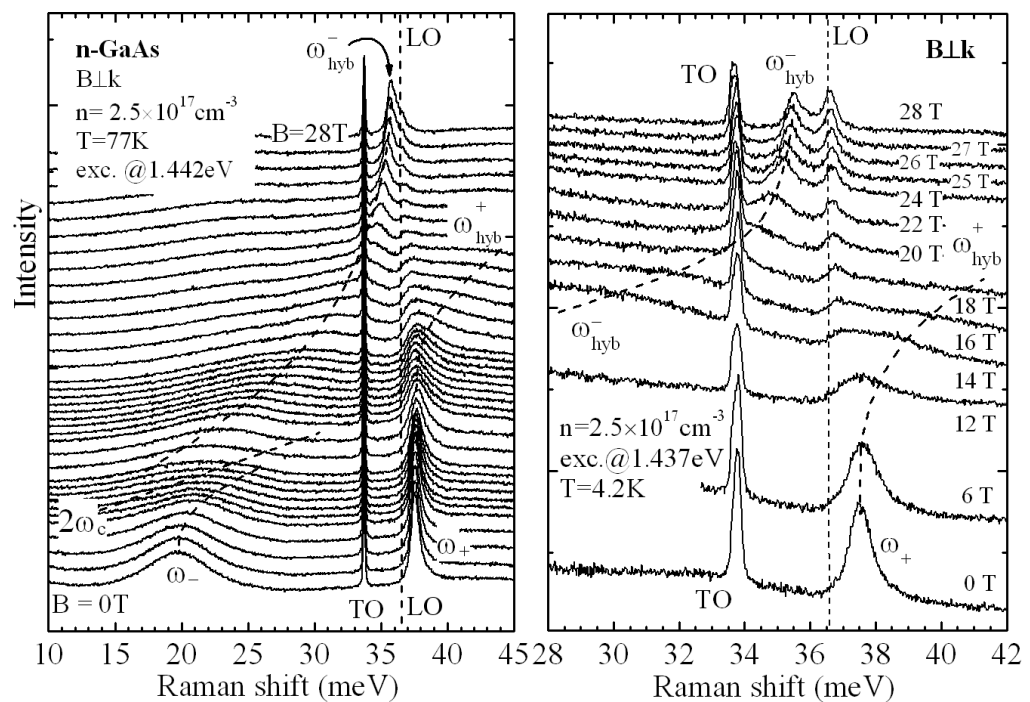

Fig. 5. Voigt configuration Raman scattering spectra measured as a function of the magnetic field for a sample with $n=2.5 \times 10^{17} \mathrm{~cm}^{-3}$, at $T=77 \mathrm{~K}$ (left part) and $T=4.2 \mathrm{~K}$ (right part).

Within the standard approach presented in the preceding section, this additional Raman scattering signal cannot be attributed to any longitudinal magnetoplasmon-phonon mode of the doped material. Although one could question the validity of the conventional dielectric function approach to explain the high field data, the appearance of this additional mode could be explained in terms of disorder-induced activation of the transverse plasmon-phonon modes [26]. Indeed, the appearance of the additional modes is favoured in samples with lower electron concentrations and at lower temperatures, thus in conditions for which 
stronger influence of disorder could be expected. The mechanism that breaks the usual selection rules and allows the observation of the transverse plasmon-phonon modes is not completely clear and needs to be clarified, both experimentally and theoretically.

\section{Magnetic field induced metal-insulator transition and magnetopolaron effect}

In this section the Raman scattering spectra were obtained for samples with room temperature electron concentration which is slightly higher than the Mott transition limit. The Raman spectra of these samples show a quantitatively different behaviour than those with higher concentration, when measured at low temperatures. For such samples, both the temperature decrease and the increase in the magnetic field strength induce carriers' localization, which results in a decrease in plasma density. This allows the observation how the Raman signals corresponding to collective plasma excitation undergo gradual transformation to single particle excitations of electrons localized at donor centres.

Let us focus on the sample which shows changes in the electron concentration as a function of temperature, from $n \approx 6 \times 10^{16} \mathrm{~cm}^{-3}$ at $T=77 \mathrm{~K}$ to $n \approx$ $3.8 \times 10^{16} \mathrm{~cm}^{-3}$ at $4.2 \mathrm{~K}$, as can be estimated from the measured energy position of coupled plasmon-phonon modes. The representative spectra are shown in Fig. 6 . The zoomed part of these spectra around the energy of the undressed LO-phonon is presented in the right part of Fig. 6 .

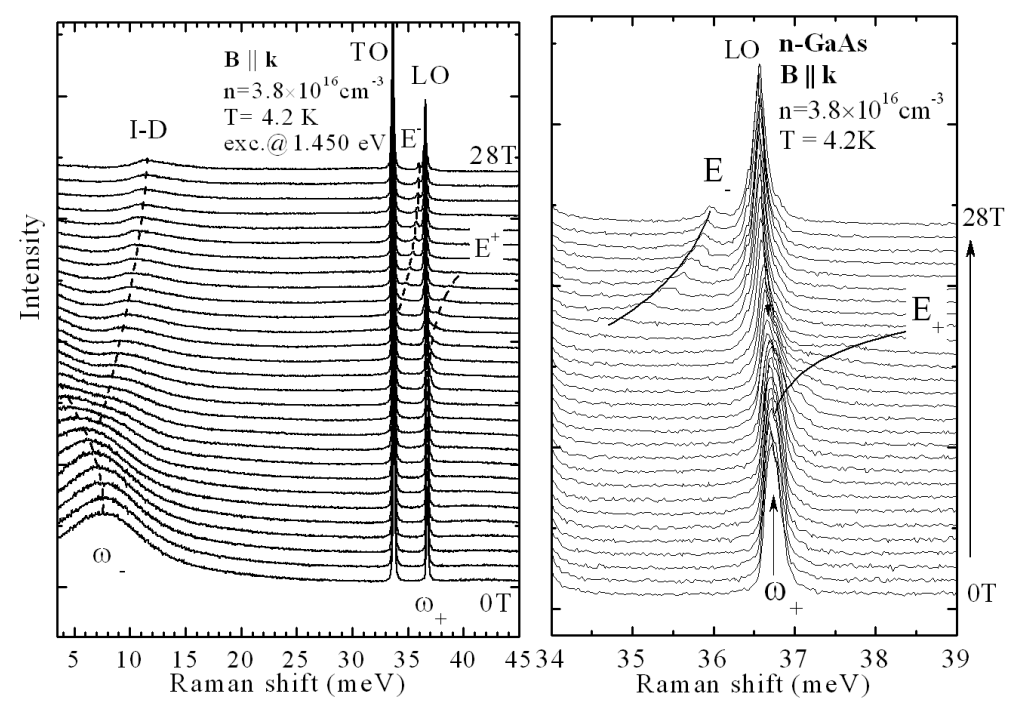

Fig. 6. Magnetic field evolution of Raman scattering spectra measured in Faraday configuration at $T=4.2 \mathrm{~K}$ for a sample with $n=3.8 \times 10^{16} \mathrm{~cm}^{-3}$ in the broad energy range (left part). Right part: the zoom of the data around LO-phonon energy. 
In this sample, the magnetic field noticeably affects the Faraday configuration spectra, in contrast to the case of previously discussed results for samples with a higher electron concentration. The characteristic $\omega^{-}$and $\omega^{+}$plasmon-phonon modes are still well defined in the spectra measured at zero magnetic field. However, both $\omega^{-}$and $\omega^{+}$peaks shift toward lower energies, progressively when the magnetic field is applied. The energy of $\omega^{-}$tends to decrease to zero and merges with the laser line position whereas $\omega^{+}$approaches the position of the undressed LO-phonon line at sufficiently high magnetic fields. In addition, new signals appear at high magnetic fields: one in the low energy part of the spectra denoted as $I-D$ in Fig. 6 and two others, $E^{+}$and $E^{-}$in the vicinity of the LO-phonon peak (see the left part in Fig. 6). The evolution of the energy positions of the measured Raman scattering signals versus magnetic field is shown in detail in Fig. 7.

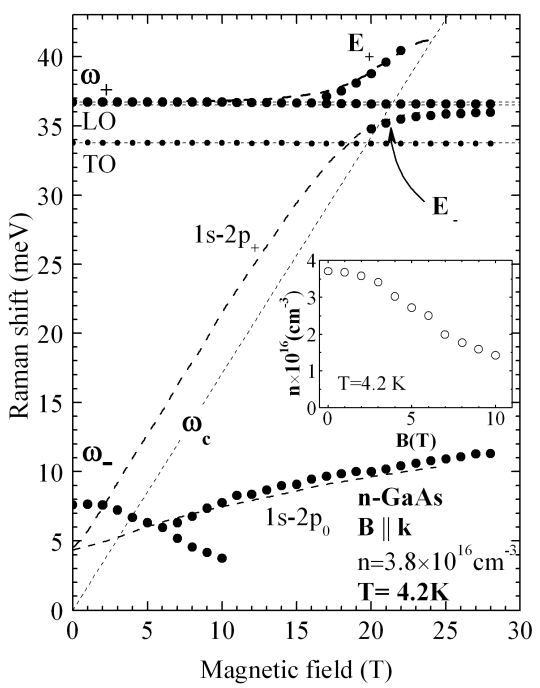

Fig. 7. Magnetic field dependence of peak positions of Raman scattering signal measured in Faraday configuration at $T=4.2 \mathrm{~K}$ for a sample with $n=3.8 \times 10^{16} \mathrm{~cm}^{-3}$.

The appearance of the effect of the magnetic freeze-out is essential to interpret these data. The electron concentration can be reasonably well monitored by the position of the $\omega^{-}$peak, and the estimated changes of $n$ as a function of the magnetic field are shown in the inset to Fig. 7. The logical consequence of the freezing of electrons on donor sites is the appearance of the Raman signal related to intra-donor transitions. The additional transitions which are observed in the spectra at high magnetic field are attributed to the Raman scattering signals related to the excitations between the $1 s$ ground state and the excited states of electrons frozen on donor states. The $I-D$ line is assigned to the $1 s-2 p_{0}$ transition, which is active in the Raman scattering processes and usually observed in experiments on insulating $n$-type semiconductors [25]. The $E^{+}$and $E^{-}$peaks are 
most likely associated with the $1 s-2 p^{+}$transition in the region, where it splits due to the resonant magnetopolaron effect [26]. Usually, the $1 s-2 p^{+}$transition does not contribute to the Raman scattering signal, but presumably it becomes active when mixed with the LO-phonon mode due to the Fröhlich interaction. Both $1 s-2 p^{+}$and $1 s-2 p_{0}$ transitions can be, however, investigated in far-infrared magnetoabsorption experiments, in a wide range of magnetic fields. In Fig. 7 the positions of the $1 s-2 p^{+}$and $1 s-2 p_{0}$ transitions measured in the Raman scattering experiments is compared with those reported previously from far-infrared absorption studies on $n$-type GaAs [10]. Dashed lines in Fig. 7 represent the overlapping theoretical and experimental results which have been digitized from Ref. [10]. These lines also coincide well with the Raman data, which confirms the presented interpretation. It is worth noticing that, similar to far-infrared magnetoabsorption experiments on different $n$-type semiconductor structures, we observe the effect of resonant magnetopolaron coupling when electrons are bound to donors (interaction between $1 s-2 p^{+}$and LO-phonon excitations), but we see hardly any traces of resonant Fröhlich interaction in the metallic phase, between the cyclotron-resonance and any other LO-phonon-like modes. This observation may also give some insight into the physics of the resonant magnetopolaron effect, which has been recently reviewed in two-dimensional systems [30-32]. Besides, the presented results show an interesting possibility of studying the metal-insulator transition in $n$-type semiconductors using the Raman scattering experiments.

\section{Conclusions}

The Raman scattering spectra of $n$-type GaAs samples show prominent features due to coupled plasmon-LO-phonon modes. In samples with the highest electron concentration the observed evolution of the Raman scattering spectra with the magnetic field is consistent with a classical approach. The conventional understanding of high field Raman scattering spectra breaks down in samples with progressively lower electron concentration. The first effect of lowering electron concentration is the appearance, at high magnetic fields, of a Raman scattering signal in a very close vicinity of the LO phonon of the undoped material. This peak is tentatively assigned as due to a disorder activated transverse plasmon-phonon excitation.

The field induced metal-insulator transition is reflected in the Raman scattering experiments on samples with the lowest electron concentrations. The plasmon-phonon modes characteristic of the metallic state are replaced by intradonor excitations in the case of the insulating state. The resonant magnetopolaron effect reflecting the interaction between the LO-phonon and the $1 s-2 p$ intradonor excitation is observed in the insulating state but no coupling between LO-phonon and cyclotron resonance excitations is seen in the metallic state. 


\section{Acknowledgments}

The author is very grateful to M. Potemski for stimulating ideas and instantaneous discussions, T. Słupiński and Z. Żytkiewicz for support with samples and D. Plantier for the common experimental work. This work was partially supported by European Commission from the 6th framework programme "Transnational Access - Specific Support Action", contract RITA-CT-2003-505474 and Alexander von Humboldt Foundation.

\section{References}

[1] P.M. Platzman, P.A. Wolff, Waves and Interactions in Solid State Plasmas, Academic Press, New York 1973.

[2] A. Mooradian, G.B. Wright, Phys. Rev. Lett. 16, 999 (1966).

[3] B.B. Varga, Phys. Rev. 137, A1896 (1965).

[4] A. Pinczuk, G. Abstreiter, R. Trommer, M. Cardona, Solid State Commun. 21, 959 (1977).

[5] G. Abstreiter, R. Trommer, M. Cardona, A. Pinczuk, Solid State Commun. 30, 703 (1979).

[6] U. Nowak, W. Richter, G. Sachs, Phys. Status Solidi B 108, 131 (1981).

[7] G. Abstreiter, M. Cardona, A. Pinczuk, in: Light Scattering in Solids IV, Eds. M. Cardona, G. Guntherodt, Springer-Verlag, Berlin 1984, p. 68.

[8] P.M. Platzman, P.A. Wolff, N. Tzoar, Phys. Rev. 174, 489 (1968).

[9] B. Lax, J. Phys. Soc. Jpn. 21, 165 (1966).

[10] J.P. Cheng, B.D. McCombe, J.M. Shi, F.M. Peeters, J.T. Devreese, Phys. Rev. $B$ 48, 7910 (1993).

[11] M. Grynberg, S. Huant, G. Martinez, J. Kossut, T. Wojtowicz, G. Karczewski, J.M. Shi, F.M. Peeters, J.T. Devreese, Phys. Rev. B 54, 1467 (1996).

[12] R. Kaplan, E.D. Palik, R.F. Wallis, S. Iwasa, E. Burstein, Y. Sawada, Phys. Rev. Lett. 18, 159 (1967).

[13] S. Ivasa, in: Physics of Solids in Intense Magnetic Fields, Ed. E.D. Haidemenakis, Plenum Press, New York 1969, p. 126.

[14] I. Yokota, J. Phys. Soc. Jpn. 21, 738 (1966).

[15] E.D. Palik, J.K. Furdyna, Rep. Prog. Phys. 33, 1193 (1970).

[16] B. Zhang, M.F. Manger, E. Batke, Phys. Rev. Lett. 89, 039703 (2002).

[17] A.J.L. Poulter, C. Faugeras, J. Zeman, M. Potemski, D.K. Maude, G. Martinez, Phys. Rev. Lett. 89, 039704 (2002).

[18] C.K. Patel, R.E. Slusher, Phys. Rev. Lett. 21, 1563 (1968).

[19] N. Tzoar, E. Foo, Phys. Rev. 180, 535 (1969).

[20] L.V. Kulik, I.V. Kukushkin, V.E. Kirpichev, K. v. Klitzing, K. Eberl, Phys. Rev. $B$ 61, 12717 (2000), and references therein.

[21] V.E. Kirpichev, L. v. Kulik, I.V. Kukushkin, K. v. Klitzing, K. Eberl, W. Wegscheider, Phys. Rev. B 59, R12751 (1999). 
[22] A.J.L. Poulter, J. Zeman, D.K. Maude, M. Potemski, G. Martinez, A. Riedel, R. Hey, K.J. Friedland, Phys. Rev. Lett. 86, 336 (2001), and references therein.

[23] S.N. Klimin, J.T. Devreese, Phys. Rev. B 68, 245303 (2003).

[24] L. Wendler, R. Pechstedt, J. Phys., Condens. Matter 2, 8881 (1990).

[25] A. Wysmolek, Phys. Status Solidi B 235, 48 (2003).

[26] A. Wysmolek, D. Plantier, M. Potemski, T. Słupiński, Z.R. Żytkiewicz, Phys. Rev. B 74, 165206 (2006).

[27] A. Wysmolek, M. Potemski, T. Slupinski, Physica B 298, 216 (2001).

[28] R. Kaplan, E.D. Palik, R.F. Wallis, S. Iwasa, E. Burstein, Y. Sawada, Phys. Rev. Lett. 18, 159 (1967).

[29] I.B. Bernstein, Phys. Rev. 109, 10 (1958).

[30] C. Faugeras, G. Martinez, A. Riedel, R. Hey, K.J. Friedland, Yu. Bychkov, Phys. Rev. Lett. 92, 107403 (2004).

[31] C. Faugeras, G. Martinez, Yu. Bychkov, Phys. Rev. Lett. 94, 239702 (2005).

[32] S.N. Klimin, J.T. Devreese, Phys. Rev. Lett. 94, 239701 (2005). 